\title{
Corruption and competition in procurement
}

\author{
Marco Celentani ${ }^{\mathrm{a}, *}$, Juan-José Ganuza ${ }^{\mathrm{b}}$ \\ ${ }^{a}$ Department of Economics, Universidad Carlos III de Madrid, Getafe, \\ Madrid 28903, Spain \\ ${ }^{\mathrm{b}}$ Department of Economics and Business, Universitat Pompeu Fabra, Carrer Ramon Trias \\ Fargas 25-27, 08005 Barcelona, Spain
}

\begin{abstract}
We consider a procurement problem in which the procurement agent is supposed to allocate the realization of a project according to a competitive mechanism that values bids in terms of the proposed price and quality. Potential bidders have private information about their production costs. Since the procurement agent is also in charge of verifying delivered quality, in exchange for a bribe, he can allow an arbitrary firm to be awarded the realization of the project and to produce a quality level lower than that announced. We compute equilibrium corruption and we study the impact on corruption of the competitiveness of the environment, and in particular of: (i) an increase in the number of potential suppliers of the good or service to be procured, and (ii) an increase of competition in the market for procurement agents. We identify the effects that influence equilibrium corruption and show that, contrary to conventional wisdom, corruption may well be increasing in competition.
\end{abstract}

JEL classification: C72; D73; H57; K42

Keywords: Corruption; Competition; Public procurement

Toute institution qui ne suppose pas que le peuple est bon, et le magistrat corruptible, est vicieuse.

Maximilien de Robespierre (1793)

Project de déclaration de droits de l'homme.

\footnotetext{
${ }^{*}$ Corresponding author. Fax: +34-91-624-98-75.

E-mail address: celentan@eco.uc3m.es (M. Celentani).
} 


\section{Introduction}

Corruption has often been argued to exist because the lack of competition generates rents that can be illegally appropriated. This general idea has often led people into thinking that, since increased competition reduces rents, it also leads to lower corruption. Rose-Ackerman (1996) maintained that 'In general any reform that increases the competitiveness of the economy helps reduce corrupt incentives'.

The goal of this paper is to provide a positive theoretical analysis of the impact of competition on corruption and show that there are reasons to doubt that increasing the competitiveness of the environment is guaranteed to lead to reduced corruption.

We consider a procurement problem and we focus our attention on a situation in which corruption is likely to prosper, i.e., a case in which the good to be procured is not homogeneous but can be produced at different quality levels and in which the agent has superior information about delivered quality. The previous assumptions imply that, in exchange for a bribe, the agent can assign the project to a firm he favors and hide the fact that it delivers lower quality than promised.

Taking advantage of existing results on multidimensional mechanism design, we characterize equilibrium corruption and study how it depends on the degree of competitiveness of the environment. We identify the effects through which higher competition affects corruption and find that, contrary to conventional wisdom, the total effect is everything but clear cut: more competition may lead to both higher or lower corruption.

To be more specific, we consider the impact of increased competition both in the procurement market and in the market for procurement agents. In the former case we show that an increase in the number of potential firms has effects with different signs and that the net impact is ambiguous. We show that for specific functional forms the net effect of the increase in the number of potential suppliers may well be to increase corruption. In the case of the market for procurement agents we argue that, if increased competition implies that the employed procurement agent is more efficient in verifying delivered quality, the optimal mechanism will increase corruption profitability and corruption will unambiguously be higher.

In terms of modeling choices our paper is closely related to the extensive literature on optimal procurement mechanisms and in particular to Laffont and Tirole (1991), who explicitly consider the impact of corruption. Since we are interested in the comparative statics of the competitiveness of the environment, we make use of Che's (1993) characterization of optimal mechanisms to generalize the results of Laffont and Tirole (1991) to a case in which procurement agents and firms are heterogeneous in terms of continuous parameters. 
While in this paper we have chosen to abstract away from dynamic considerations, in Celentani and Ganuza (1999) we take the view that it is important to also study the dynamics of corruption. To do this we embed a simplified version of the setting described in this paper into an infinite horizon framework in which two generations of agents overlap at each date and we study the dynamic response of corruption to both temporary and permanent shocks to the profitability of corruption. In another related paper (Celentani and Ganuza, 2001), we study the impact on equilibrium of the degree of decentralization of corruption decisions by analyzing the consequences of allowing agents to coordinate their corruption decisions in such a way as to maximize their total proceeds.

In terms of goals our paper should be seen as a contribution to a recent strand of literature that studies the ways in which the degree of competitiveness of the environment affects corruption. Bliss and Di Tella (1997) argue that competition also depends on corruption as the latter affects firms' entry decisions and show that the effects of increased competition on corruption are generally ambiguous. Laffont and N'Guessan (1999) consider a model of regulation in which the agent may be corrupt and in which the regulator optimally chooses the contract to be offered to the regulated firm and to the agent. Increased competition among agents leading to a better monitoring technology is seen to enlarge the set of parameters for which corruption may result in equilibrium - a conclusion similar to our result on increased competition in the market for procurement agents. Ades and Di Tella (1999) propose a simple model in which a regulation agent may be corrupt and argue that an increase in competition parameters that lower regulated firms' equilibrium profits has an ambiguous effect on the level of corruption due to the regulator's optimal response that changes the agent's wage and therefore his incentives to be corrupt. Wei's (2000) simple theoretical model suggests that countries with higher 'natural openness', i.e., exogenous factors favoring openness to trade, have more incentives to keep corruption low.

The relationship between competition and corruption has been the object of several recent cross-sectional studies. Ades and Di Tella (1997) find that an active industrial policy increases corruption. Ades and Di Tella (1999) show some support for the idea that higher levels of openness to trade are associated to lower corruption levels. Laffont and N'Guessan (1999) find some support for the same relationship on African data, but when they also include lagged corruption as a regressor to account for the interdependence of corruption and competition they find that corruption is decreasing in the openness of the economy for low historical corruption levels and increasing otherwise. Wei (2000) shows that 'natural openness' explains $60 \%$ of cross-sectional variation in corruption while residual openness factors have no significant effect on corruption. 
While the previous papers can be regarded as providing some support for a negative relationship between competition and corruption, we believe that the ambiguity of some of the results of our paper indicates that the sign of this relationship cannot be determined at the aggregate level. In different words, our results suggest that the initial evidence on a negative association between competition and corruption discussed above should not be taken to mean that any increase in competition is guaranteed to reduce corruption.

The paper is structured in the following way. Section 2 presents the model. In Section 3 the optimal mechanism for the procurement process is derived keeping into account a given probability of corruption and it is shown to amount to the application of a discretion index. Section 4 derives the payoff from corrupt behavior and explains how corruption decisions are taken in the face of a given discretion level. Section 5 combines the previous two sections to obtain equilibrium corruption. Section 6 presents the main results of the paper by analyzing how corruption varies with the competitiveness of the environment. Section 7 concludes.

\section{The model}

Consider a government agency that has to procure a good that is not homogeneous but can be produced according to different specifications that can be summarized by a quality level, $q$. The good can be produced by any one of $N$ potential suppliers which have private information about their own costs of producing quality level $q$. In the following we will assume that the cost function of firm $i=1, \ldots, N$ is

$$
C^{i}(q)=C\left(q, \theta_{i}\right)=\theta_{i} q,
$$

where $\theta_{i}$ is to be interpreted as the constant marginal cost of quality which is known only to firm $i$. Firms are risk-neutral, $U_{i}^{F}(p, q)=p-C^{i}(q)$, and maximize expected profit. Suppose that the administrator of the agency (in the following, the principal) is also risk neutral and his utility is increasing (with diminishing marginal utility) in quality and decreasing in the (gross) transfer to the supplier, $U^{P}(p, q)=V(q)-p$; for concreteness we will work with the specific functional form $U^{P}(p, q)=\log q-p$.

This kind of situations has been studied by several authors and the optimal allocation mechanism has been shown to be a multidimensional one that values both price and quality, ${ }^{1}$ - a type of mechanism that is employed very frequently by many procurement agencies. ${ }^{2}$

\footnotetext{
${ }^{1}$ See, for example, Che (1993), Laffont and Tirole (1991) and Branco (1997).

${ }^{2}$ Che (1993) and Laffont and Tirole (1991) refer to examples in which mechanisms are used that award points to price and other parameters (such as technical characteristics and delivery dates) and that have been used by the US Department of Defense and US electric utilities.
} 
Suppose now that, while the principal can set the guidelines for the procurement process by announcing the allocation mechanism that is to be employed, he is then forced to delegate the execution of the procurement process to an agent who has superior information on delivered quality. While giving weight to quality has the desirable efficiency properties described above, it obviously opens up the opportunity for the agent to be corrupt and favor a particular firm bidding a high price on the grounds that it offers a technically superior good.

To describe how the possibility of the agent being corrupt modifies the problem we find it convenient to summarize the extensive form as follows.

1. Nature:

(a) Selects the procurement agent's idiosyncratic cost of becoming corrupt, $\beta$. It is common knowledge that $\beta$ is drawn from the uniform distribution on $[0, \bar{\beta}]$.

(b) Selects the marginal costs of quality $\theta_{i}$ for firms $i=1, \ldots, N$. It is common knowledge that each $\theta_{i}$ is identically and independently drawn from the uniform distribution on $[\underline{\theta}, \bar{\theta}]$, i.e.,

$f(\theta)=1 /(\bar{\theta}-\underline{\theta}), \quad F(\theta)=(\theta-\underline{\theta}) /(\bar{\theta}-\underline{\theta})$.

2. The procurement agent privately learns his idiosyncratic cost of becoming corrupt, $\beta$. Each firm privately learns its marginal cost of producing quality, $\theta_{i}$.

3. The procurement agent decides whether to be honest or corrupt. If he decides to be corrupt, he pays the (sunk) cost $\beta$ in order to establish a network of contacts that will then enable him to arrange corrupt transactions.

4. Without observing firms' marginal costs, the agent's idiosyncratic corruption $\operatorname{cost} \beta$, or his decision to be corrupt or not, the principal publicly announces the mechanism to be used.

5. Execution of the procurement process:

$\mathrm{H}$. If the agent decided to be honest, he manages the mechanism honestly. Firms participate in the mechanism and the winning firm is selected, it receives a transfer and produces the good at a specific quality level $q$. The agent verifies that delivered quality is $q$ and certifies it. $^{3}$

\footnotetext{
${ }^{3}$ While the extent to which realized quality might differ from planned quality as a consequence of random factors might depend on the specific project, the assumption that, conditional on the agent being honest, no moral hazard problem exists between the agent and the firms is made only for expositional convenience. McAfee and McMillan (1987) have shown that in a model with moral hazard but otherwise identical to ours the optimal contract awards the firm a payment that is linear in realized quality. Allowing for the agents to imperfectly observe realized quality would only make notation heavier and would not change any of the results of the paper.
} 
C. If the agent decided to be corrupt:

(a) $\mathrm{He}$ is randomly matched to one firm $i$, learns its marginal cost $\theta_{i}$, and demands it a bribe $b$ to be received in exchange for the agreement that the firm will be awarded the project under conditions to be detailed and will be permitted to produce a lower quality, $q_{C}$.

(b) The firm accepts or rejects.

A. If the firm accepts, all firms participate in the mechanism, but the firm that was matched with the agent is selected as the winner under the claim that it is the firm that, according to the allocation mechanism, offers the best quality/price combination; the firm receives a transfer, produces the good at a lower quality level than specified by the mechanism, $q_{C}<q$, and pays out the bribe $b$ to the agent.

The agent verifies that delivered quality is $q_{C}$ and certifies it is the one specified by the allocation mechanism, $q$.

With probability $\mu$ the corrupt agreement is detected and in this case the procurement agent and the firm awarded the project are imposed penalties, $B^{A}>0$ and $B^{F}>0$, respectively. ${ }^{4}$

R. If the firm rejects, the agent has no additional opportunity to contact another firm and therefore manages the mechanism honestly.

Firms participate in the mechanism and the winning firm is selected, it receives a transfer and produces the good at a specific quality level $q$. The agent verifies that delivered quality is $q$ and certifies it.

To conclude the description we only need to comment on the agent's preferences. The agent is assumed to be risk-neutral and we normalize his wage to 0 so that the agent's expected income will be 0 unless he plans to be corrupt in which case it will be equal to the expected bribe minus the idiosyncratic corruption cost $\beta$ minus the expected penalty. ${ }^{5}$

In the following subsection we discuss our modeling choices.

\subsection{Discussion of the model}

In our model we assume that the procurement agent has to decide whether to be corrupt or not before he observes the mechanism chosen by the principal. This assumption together with Assumption 2 below, guaranteeing that, once the corruption cost $\beta$ is sunk, corruption always pays off, implies that

\footnotetext{
${ }^{4}$ Since the principal's preferences over final outcomes discussed above are independent of the penalties, these are best thought as non-pecuniary penalties or pecuniary penalties not accruing to the principal.

${ }^{5}$ Since it is standard to assume that an agent who is found to be corrupt is deprived at least part of his salary, what turns out to be relevant is not the wage the agents gets but the premium over the wage in alternative employment opportunities. Since the agent is risk neutral, assuming a positive wage premium is equivalent to having a positive expected penalty. This implies that normalizing the wage premium to 0 has no impact on qualitative results.
} 
the principal has no ability to actively influence the agent's decision to be corrupt or not, and that he regards it as given.

In an alternative formulation the principal could be allowed to play as a leader and commit to using a certain allocation mechanism prior to the agent deciding whether to be corrupt or not. In this way the principal would no longer regard the corruption decision as a given, but would try to influence it in such a way as to maximize his expected surplus.

We view the two setups as two extremes. While we only consider an irreversible decision to be corrupt or not, the alternative formulation describes a situation in which the decision to be corrupt has no element of irreversibility whatsoever - an agent has enough time to decide whether to be corrupt or not after the awarding mechanism has been announced and before the project is awarded.

The reasons we consider the current setup as the appropriate modeling choice are the following: ${ }^{6}$

1. Assuming that the decision to be corrupt is taken before observing the mechanism chosen by the principal translates to a static framework the idea that the decision to be corrupt has an element of irreversibility. By this we mean that an agent who decides to be corrupt typically sinks a fixed cost to arrange a network of contacts that can then be used for several transactions. If this is true, the extent to which the principal can influence the agent's final choice to be corrupt or not in a particular transaction may be very limited.

2. Given the nature of the problem, we think of the principal not as a legislator who can credibly set procurement mechanisms rules by decree, but as an administrator of a procurement agency who, though benevolent, is in charge for a relatively short period and therefore can do no (much) better than optimally respond to a decision to be corrupt that is (largely) independent of the mechanism he will choose to employ.

3 . We regard our paper as giving a positive rather than a normative contribution and we therefore believe it is important not to overestimate the commitment power of public administrators.

Other assumptions of the extensive form (the agent learns the type of the firm before making a bribe demand and he is endowed with all the bargaining power) are made only for the sake of simplicity and are in no way essential to derive any of our results.

It is also important to stress that we have chosen to specify functional forms for utility functions and distribution functions to simplify the presentation. As will be clear later on, the qualitative results we present below can be

\footnotetext{
${ }^{6} \mathrm{We}$ want to thank an anonymous referee for pointing out to us the importance of discussing the alternative formulations above.
} 
generalized provided that the characterization and the implementation of the optimal mechanism we present are valid. Section 2 of Che (1993) presents sufficient conditions on $V(\cdot), C(\cdot, \cdot)$ and the distribution of firms' types for this to be true. ${ }^{7}$

\section{Optimal mechanisms with corruption}

This section studies the optimal mechanism for a given probability of the agent to be corrupt. We first study the optimal procurement mechanism when the agent is not corrupt. We then specify the outcome of a corrupt transaction. Finally, we provide the optimal mechanism for a given probability for the agent to be corrupt.

\subsection{The auction with an honest agent}

An honest agent simply manages the mechanism he is instructed to employ and allocates the project accordingly. This observation lets us establish a reference point adapting to our setting a result of Che (1993), that is itself a special case of results of Laffont and Tirole (1987), McAfee and McMillan (1987), and Riordan and Sappington (1987). The proof of Proposition 1 is nevertheless presented in the appendix as it will be later used in the proof of Proposition 4.

Proposition 1. In the optimal mechanism, the firm with the lowest $\theta$ is selected and it produces quality $q_{0}$ maximizing $V(q)-\theta_{q}-(\theta-\underline{\theta}) q=\log q-$ $(2 \theta-\underline{\theta}) q$.

Proof. See the appendix.

The interpretation of the above proposition is that in the optimal mechanism the marginal cost of quality is overvalued ( since $2 \theta-\underline{\theta} \geqslant \theta$ ), or equivalently that the marginal value of quality is undervalued to reduce the informational rents.

The next issue to discuss is the implementation of the optimal mechanism. An analogy with unidimensional auctions naturally focuses attention on the so-called first-score auctions, i.e., auctions in which offers, quality - price pairs, are evaluated according to a prespecified 'score' function, the winner is selected to be the firm that offered the highest score combination, is required to produce the quality it offered and is paid the price it bid. In the following

\footnotetext{
${ }^{7}$ The assumption that $\beta$ is uniformly distributed, on the other hand is in no way essential.
} 
we will show that a first-score auction with an appropriate score function implements the optimal mechanism. ${ }^{8}$

We start by characterizing the equilibrium of first-score auction when the score function is

$$
\tilde{S}(\lambda, q, p)=\lambda \log q+\frac{\theta}{2} q-p .
$$

Proposition 2. Under the scoring rule $\tilde{S}(\lambda, q, p)=\lambda \log q+(\underline{\theta} / 2) q-p$, firms' symmetric equilibrium offers in the first-score auction $\left(q_{\mathrm{E}}\left(\theta_{i}, \lambda\right), p_{\mathrm{E}}\left(\theta_{i}, \lambda\right)\right)$ are

$$
\begin{aligned}
& q_{\mathrm{E}}\left(\theta_{i}, \lambda\right)=\frac{2 \lambda}{2 \theta_{i}-\underline{\theta}}, \\
& p_{\mathrm{E}}\left(\theta_{i}, \lambda\right)=\frac{2 \lambda \theta_{i}}{2 \theta_{i}-\underline{\theta}}+\int_{\theta_{i}}^{\bar{\theta}}\left(\frac{\bar{\theta}-s}{\bar{\theta}-\theta_{i}}\right)^{N-1} \frac{2 \lambda}{2 s-\underline{\theta}} \mathrm{d} s .
\end{aligned}
$$

Proof. Immediate from Che (1993).

The following corollary provides an additional characterization of the equilibrium of the above first-score auction.

Corollary 1. Under the scoring rule $\tilde{S}(\lambda, q, p)=\lambda \log q+(\underline{\theta} / 2) q-p$, the expected payoff of the winning firm and firms' equilibrium bid price and quality for all $\theta \in[\underline{\theta}, \bar{\theta}]$ are increasing in $\lambda$.

Proof. Immediate.

The corollary shows that a higher value of $\lambda$, i.e., a higher weight given to quality in the scoring rule, implies that in equilibrium firms will offer higher levels of quality together with higher prices but also that the higher value given to quality will increase the informational rents of the firms with lower marginal costs of producing quality, therefore guaranteeing the winning firm a higher margin over costs.

\footnotetext{
${ }^{8}$ Another class of mechanisms, second-score auctions, differ from first score auctions in that the winner is allowed to choose its preferred price - quality combination among the ones that have the same score as the second highest score offer. Second-score auctions are known to implement the optimal mechanism if the same score function is used that makes the first-score-auction optimal. For details, see Che (1993).
} 
In the following, let $\theta_{1}=\min \left\{\theta_{i}\right\}_{1=1}^{N}$ denote the first-order statistic of $\left\{\theta_{i}\right\}_{1=1}^{N}$ (i.e., the minimum over the sample of size $N$ ), let

$$
M_{N}\left(\theta_{1}\right)=1-\left(1-F\left(\theta_{1}\right)\right)^{N}=1-\left(\frac{\bar{\theta}-\theta_{1}}{\bar{\theta}-\underline{\theta}}\right)^{N}
$$

denote its distribution function and let

$$
\begin{aligned}
& Q=\int_{\underline{\theta}}^{\bar{\theta}}\left[\frac{2}{2 \theta_{1}-\underline{\theta}}\right] \mathrm{d} M_{N}\left(\theta_{1}\right), \\
& P=\int_{\underline{\theta}}^{\bar{\theta}}\left[\frac{2 \theta_{1}}{2 \theta_{1}-\underline{\theta}}+\int_{\theta_{1}}^{\bar{\theta}}\left(\frac{\bar{\theta}-s}{\bar{\theta}-\theta_{1}}\right)^{N-1} \frac{2}{2 s-\underline{\theta}} \mathrm{d} s\right] \mathrm{d} M_{N}\left(\theta_{1}\right) .
\end{aligned}
$$

Corollary 2. (1) Under the scoring rule $\tilde{S}(\lambda, q, p)$ the expected winning offer of a first-score auction $\left(q^{*}(\lambda), p^{*}(\lambda)\right)$ is

$$
\begin{aligned}
& q^{*}(\lambda)=E_{\theta_{1}}\left[q_{\mathrm{E}}\left(\theta_{1}, \lambda\right)\right]=\int_{\underline{\theta}}^{\bar{\theta}} q_{\mathrm{E}}\left(\theta_{1}, \lambda\right) \mathrm{d} M_{N}\left(\theta_{1}\right), \\
& p^{*}(\lambda)=E_{\theta_{1}}\left[p_{\mathrm{E}}\left(\theta_{1}, \lambda\right)\right]=\int_{\underline{\theta}}^{\bar{\theta}} p_{\mathrm{E}}\left(\theta_{1}, \lambda\right) \mathrm{d} M_{N}\left(\theta_{1}\right) .
\end{aligned}
$$

(2) Expected winning price and quality are positive linear functions of $\lambda, q^{*}(\lambda)=Q \lambda, p^{*}(\lambda)=P \lambda$.

Proof. See the appendix.

The following proposition that adapts a result in Che (1993) shows that when the score function is $\tilde{S}(\lambda, q, p)$ the first-score auction implements the optimal mechanism, provided that $\lambda=\frac{1}{2}$.

Proposition 3. Under the scoring rule $\tilde{S}(\lambda, q, p)=\lambda \log q+(\underline{\theta} / 2) q-p$, with $\lambda=\frac{1}{2}$, the first-score auction implements the optimal mechanism.

Proof. Immediate from Che (1993).

The interpretation of Proposition 3 is the same as that of Proposition 1. The score function underestimates the marginal value of quality with respect to money to limit the informational rents of relatively efficient firms. 


\subsection{A corrupt agent}

When the procurement agent decides to be corrupt he sinks fixed cost $\beta$. After the principal announces the mechanism to be used, the corrupt agent is matched with a random firm, learns its type and makes it a take-it-or-leave-it bribe demand in exchange for replacing ex-post its bid with one that guarantees it to be awarded the project with probability 1 and with the understanding that the firm will be allowed to produce an exogeneously given minimal level of quality $q_{C},{ }^{9}$ lower than the one stated in the winning bid. ${ }^{10}$

While it is realistic to assume that the agent has some leeway in manipulating bids, it is also reasonable to assume that its extent is limited as announcing offers with low prior probability might trigger investigations. We model the agent's ability to manipulate the bid of firm $i$, by assuming that the replaced bid will be the equilibrium bid of a type

$$
\theta^{\prime}=\min _{j \neq i} \theta_{j}-\phi\left(\min _{j \neq i} \theta_{j}\right),
$$

where $\phi(\cdot)$ satisfies the following conditions:

$$
\begin{aligned}
& \phi(\theta) \geqslant 0, \quad \forall \theta \in[\underline{\theta}, \bar{\theta}], \\
& \theta-\phi(\theta) \geqslant \underline{\theta}, \quad \forall \theta \in[\underline{\theta}, \bar{\theta}], \\
& \int_{\underline{\theta}}^{\bar{\theta}} p^{*}(\theta-\phi(\theta)) \mathrm{d} M_{N-1}(\theta)=\int_{\underline{\theta}}^{\bar{\theta}} p^{*}(\theta) \mathrm{d} M_{N}(\theta),
\end{aligned}
$$

\footnotetext{
${ }^{9}$ Notice that all the qualitative results of this paper would also hold if we allowed $q_{C}$ to be increasing in the quality level of the winning firm, provided that this dependence does not imply that for higher levels of corruption, the principal prefers a higher $\lambda$.

${ }^{10} \mathrm{~A}$ procurement mechanism in the described setting allocates the realization of the project to one firm and makes transfers to possibly more than one firm. Despite the fact that in the absence of corruption there are optimal mechanisms that make payments to firms that are not called to realize the project, in the following we restrict the space of mechanisms the principal can use to mechanisms that make a transfer only to the firm that is awarded the realization of the project.

This assumption is motivated by the fact that it can be very difficult to show that a firm that does not carry out a project is not entitled to a given payment, as this would amount to showing that its true type (e.g., marginal cost) is different from the one implied by its bid. For this reason we believe that allowing mechanisms that make transfers to firms that do not carry out the project can greatly increase the possibility of corruption and this is, probably, the reason why they are so infrequent.

Since we restrict attention to mechanisms that make a payment to a firm only if this firm is called to realize the project, a corrupt agreement necessarily requires that the firm involved is awarded the project.
} 
and where $p^{*}(\theta)$ is the transfer to a firm of type $\theta$ if it is awarded the project with the mechanism chosen by the principal. In other words, the agent considers the minimum $\theta$ for firms different from firm $i$ and replaces the bid of firm $i$ with the equilibrium bid of a firm of a type that is lower than this minimum (condition (3)). Condition (4) guarantees that this bid is consistent with $\theta \in[\underline{\theta}, \bar{\theta}]$, and condition (5) guarantees that the expected transfer awarded by a corrupt agent using the proposed rule is equal to the expected transfer awarded by an honest agent.

The above conditions are meant to represent the constraints that the principal's monitoring activity imposes on the agent's behavior. Since the principal's monitoring is based on the agent's announcements, the latter cannot be statistically inconsistent with prior knowledge of bidder's marginal costs without triggering unwanted investigations.

The following assumption summarizes the behavior of an agent who decides to be corrupt. ${ }^{11}$

Assumption 1. Let $\phi(\theta)$ satisfy conditions (3)-(5). If an agent decides to be corrupt, he offers the firm a (gross) payment equal to $p^{*}(\theta-\phi(\theta), \lambda)$, where $\theta$ is the type of the firm which would have otherwise won the auction, and requires the firm to produce quality $q_{C}$.

\subsection{Optimal discretion with corruption}

When the principal knows that with probability $\gamma$ the agent is corrupt, and will therefore allocate resources in the way specified in the previous subsection, his payoff function will be

$$
(1-\gamma)(V(q)-p)+\gamma\left(V\left(q_{C}\right)-p_{C}\right)
$$

where $q_{C}$ is the exogenously fixed quality level under corruption, and $p_{C}$ denotes the transfer to the winning firm under corruption, which under Assumption 1 , is equal to $p^{*}(\theta-\phi(\theta), \lambda)$.

The next proposition generalizes Proposition 1 by characterizing the optimal mechanism when the agent is believed to be corrupt with a given probability. To do this we use the fact that, given corrupt agreements are assumed to take place before firms play according to the procurement mechanism, firms that have not been contacted by the agent play strategies that are independent of

\footnotetext{
${ }^{11}$ Assumption 1 describes the outcome of corruption given the assumption that the principal chooses a mechanism that makes transfers only to the firm that is awarded the project that was discussed in footnote 10. If the principal chose a mechanism that allows transfers to firms other than the one that is awarded the project, it would probably be reasonable to give a different description of the outcome of corruption. As was argued in footnote 10, however, the use of this kind of mechanisms in a setting in which corruption may take place seems unreasonable.
} 
the probability for the agent to be corrupt, or in other words play the same strategies as in a mechanism with an honest agent. ${ }^{12}$

Proposition 4. Under Assumption 1, in the optimal mechanism, if the agent is honest, the firm with the lowest $\theta$ is selected and it produces quality $q_{0}$ maximizing $(1-\gamma) V(q)-\theta q-(\theta-\underline{\theta}) q=(1-\gamma) \log q-(2 \theta-\underline{\theta}) q$.

Proof. See the appendix.

The following proposition shows that in order to implement the optimal mechanism with a first-score auction the principal has to set a score function whose discretion index is decreasing in the probability of the agent being corrupt:

Proposition 5. Under Assumption 1 the first-score auction with score function $\tilde{S}(\lambda, q, p)=\lambda \log q+(\underline{\theta}=2) q-p$ with $\lambda=(1-\gamma) / 2$ implements the optimal mechanism.

Proof. Straightforward from Propositions 2 and 4.

As a consequence of Proposition 4 we have:

Corollary 3. Suppose Assumption 1 is satisfied, that the agent is corrupt with probability $\gamma$ and that the principal chooses a first-score auction with the optimal scoring rule. Then, irrespective of whether the agent is honest or corrupt, expected equilibrium quality and price will be $q^{*}(\gamma)=Q(1-\gamma) / 2$ and $p^{*}(\gamma)=P(1-\gamma) / 2$.

Proof. Immediate from Proposition 5.

The above corollary formalizes Banfield's (1975) view that 'narrowing discretion [...] while preventing the agent from doing (corrupt) things that are slightly injurious to the principal it may at the same time prevent him from doing (non-corrupt) ones that would be very beneficial to him. If simply to prevent corruption an agent is given a narrower discretion than would be optimal if there were no corruption, whatever losses are occasioned by his having a sub-optimal breadth of discretion must be counted as costs of preventing corruption' (Banfield, 1975, p. 590).

\footnotetext{
${ }^{12}$ If corruption could take place after the bidding, the fact that firms know that the public official may be corrupt implies that they may want to bid in such a way as to increase the probability to be selected as partners in the corrupt transaction or in such a way as to increase their payoff if selected.
} 


\section{The decision to be corrupt}

Suppose the procurement mechanism chosen by the principal is given. If the agent decides to be honest he gets a payoff of 0 with probability 1 . If he decides to be corrupt he sinks the idiosyncratic corruption cost $\beta$, gets a bribe from the firm he is colluding with and pays a penalty if detected; the next subsection is dedicated to computing the expected payoff from being corrupt. In Section 4.2 we equate the payoffs from being honest and getting corrupt to determine for what values of $\beta$ the agent decides to be corrupt for a given procurement mechanism.

\subsection{Bargaining over a bribe}

A critical issue in the study of corruption refers to the way in which the partners of a corrupt agreement are selected and in particular to whether one should expect an efficient matching to take place or not. In our setting this concern translates to analyzing how the marginal cost of producing quality affects the surplus appropriated by the partners of a corrupt transaction via its direct influence on the cost of producing a minimally acceptable level of quality $\left(q_{C}\right)$ and its indirect influence on the firm's disagreement payoff via its expected payoff from participating in the auction. ${ }^{13}$

While the analysis of this kind of problem is complicated by the fact that corrupt transactions are arranged according to no rule but, rather, in violation of existing rules (which in turn implies that they can be organized in a variety of different ways), we believe that the illegal and therefore secret nature of corruption implies that a party potentially interested in corruption cannot rely on a public mechanism to elicit private information from all possible corruption partners and will therefore be matched with inefficient partners with positive probability.

In light of the above discussion we assume that by paying the idiosyncratic cost $\beta \in[0, \bar{\beta}]$, the agent is matched with a randomly selected firm and learns its marginal $\operatorname{cost} \theta$, or, equivalently, its willingness to enter a corrupt agreement. For the sake of simplicity as we stated in Assumption 1 we also assume that the quality the corrupt firm will have to provide is exogenously

\footnotetext{
${ }^{13}$ Some authors (e.g., Beck and Maher, 1986; Lien, 1986, 1987) consider the case in which the procurement price is given and firms bid their bribes to be awarded the project. The equilibrium of this game is equivalent to the equilibrium of the game in which firms bid procurement prices. In both cases the most efficient firm is awarded the project and equilibrium payoffs are identical. In these situations corruption only determines a different distribution of surplus between the principal and the agent. We think that these models have the drawback of not considering the optimal response of the principal and of assuming that a public mechanism, like an auction, can be used to carry out illegal transactions.
} 
given. Finally we assume the procurement agent makes a take-it-or-leave-it bribe demand to the firm. ${ }^{14}$

Given the agent makes a take-it-or-leave-it demand to the firm, the bribe he will demand is easily determined by the equality of firm's payoffs from accepting and rejecting the offer. Let $\pi_{F}(\lambda, \theta)$ denote the expected agreement payoff from a corrupt transaction to a type $\theta$ firm. This payoff is a function of its realized compensation, which, being equal to the price of the expected winning offer, is a function of the discretion index $\lambda$, and of the cost of producing quality $q_{C}$ which depends on the firm's marginal cost of producing quality $\theta$ :

$$
\pi_{F}(\lambda, \theta)=p^{*}(\lambda)-b-\theta q_{C}-\mu B^{F},
$$

or, in words, the expected price the firm gets paid minus the bribe, minus the cost of producing quality $q_{C}$, minus the expected penalty.

Let $d_{F}(\lambda, \theta)$ denote type $\theta$ firm's payoff if it rejects the agent's demand (its disagreement payoff) when discretion $\lambda$ is used in the auction. Recall that we assumed that if the firm rejects the offer of the procurement agent, the latter has no further opportunities to get corrupted and therefore manages the auction as he would have if he had decided to be honest. Given this, a type $\theta$ firm's disagreement payoff will simply be its expected payoff in an auction without corruption:

$$
d_{F}(\lambda, \theta)=\left(\lambda \int_{\theta}^{\bar{\theta}}\left(\frac{\bar{\theta}-s}{\bar{\theta}-\theta}\right)^{N-1} \frac{2}{2 s-\underline{\theta}} \mathrm{d} s\right)\left(\frac{\bar{\theta}-\theta}{\bar{\theta}-\underline{\theta}}\right)^{N-1},
$$

where the first term denotes the profit conditional upon winning the auction and the second the probability of winning the auction.

The take-it-or-leave-it equilibrium bribe can now be easily derived from the firm's participation constraint in the corruption agreement

$$
\pi_{F}(\lambda, \theta)=d_{F}(\lambda, \theta)
$$

which implies that the equilibrium bribe paid by the firm to the agent is

$$
b=p^{*}(\lambda)-\mu B^{F}-\theta q_{C}-d_{F}(\lambda, \theta)
$$

Consider now the expected payoff to the agent (gross of $\beta$ ):

$$
\pi_{A}=b-\mu B^{A} .
$$

For Eq. (6) to be an admissible solution, it is necessary that $\pi_{A}=b-\mu B^{A}>0$, or otherwise the agent prefers not to make any offer to the firm. It is easy to show that for this to happen it is necessary and sufficient that the expected surplus the agent and the firm generate once the match is established (gross

\footnotetext{
${ }^{14}$ The result is identical for any bargaining procedure that assigns a payoff to the agent that is positive and increasing in the surplus that corruption creates.
} 
of $\beta$ which is a sunk cost) but before the realization of the types of the other firms are known, is positive for all possible types of the corrupt firm:

$$
p^{*}(\lambda)-\mu\left(B^{F}+B^{A}\right)-\theta q_{C}-d_{F}(\lambda, \theta)>0 .
$$

Assumption 2 below guarantees that this condition is satisfied in the Nash equilibrium of the game.

Let

$$
A=\int_{\underline{\theta}}^{\bar{\theta}}\left[\left(\int_{\theta}^{\bar{\theta}}\left(\frac{\bar{\theta}-s}{\bar{\theta}-\theta}\right)^{N-1} \frac{2}{2 s-\underline{\theta}} \mathrm{d} s\right)\left(\frac{\bar{\theta}-\theta}{\bar{\theta}-\underline{\theta}}\right)^{N-1}\right] \frac{1}{\bar{\theta}-\underline{\theta}} \mathrm{d} \theta
$$

and

$$
\begin{aligned}
& k=\frac{1}{\bar{\beta}}\left[\frac{\bar{\theta}+\underline{\theta}}{2} q_{C}+\mu\left(B^{F}+B^{A}\right)\right], \\
& \Gamma=\frac{1}{\bar{\beta}}[(P-A)] .
\end{aligned}
$$

Assumption 2. Let $\hat{\lambda}=(1+k) /(2+\Gamma)$. For all $\theta \in[\underline{\theta}, \bar{\theta}], p^{*}(\hat{\lambda})-\mu\left(B^{F}+\right.$ $\left.B^{A}\right)-\theta q_{C}-d_{F}(\hat{\lambda}, \theta)>0$.

Section 5 shows that $\hat{\lambda}$ is the equilibrium value of discretion when Assumption 2 is satisfied. It is easy to see that, given that $\hat{\lambda}$ is bounded away from 0 and $d_{F}(\hat{\lambda}, \theta)$ tends to 0 as $N$ tends to infinity, there exist parameter values such that Assumption 2 is satisfied.

Before concluding, it is important to remark that the bribe in Eq. (6) is the bribe the agent will receive conditional on a particular type of firm and therefore not the expected bribe he anticipates when deciding whether to become corrupt, i.e., whether to be randomly matched with a firm as a potential corruption partner. The excepted payoff from corruption will be introduced in the next subsection where the agent's decision to become corrupt will be analyzed.

\subsection{The probability of corruption}

Having defined the agent's payoff when he behaves honestly and when he decides to get corrupted, we now want to compute the probability that an agent with a corruption cost $\beta$ drawn from a uniform distribution decides to get corrupted when he is supposed to allocate a project according to a first-score auction with scoring rule $\tilde{S}(\lambda, q, p)$ defined above. To do that we need to compute the probability that for a given $\lambda$ the corruption cost of the agent will be sufficiently low for him to get corrupted, or equivalently the type $\beta^{*}$ who is exactly indifferent between being honest and corrupt. In 
order to do that we first need to compute the expected payoff from becoming corrupt using the results of the previous subsection. Substituting Eq. (6) into Eq. (7) we get the agreement payoff to an agent from arranging a corrupt transaction with a firm of type $\theta$ when the discretion index of the auction is $\lambda$ :

$$
\pi_{A}(\theta, \lambda)=p^{*}(\lambda)-\mu B^{F}-\theta q_{C}-d_{F}(\lambda, \theta)-\mu B^{A} .
$$

Computing the expected value of $\pi_{A}(\theta, \lambda)$ with respect to $\theta$ we obtain the agent's expected agreement payoff when the discretion index to be used in the auction is $\lambda$. From Corollary 2 we know that $p^{*}(\lambda)=P \lambda$. The expected value of $\theta q_{C}$ is simply $(\bar{\theta}+\underline{\theta}) q_{C} / 2$ and the expected value of $d_{F}(\lambda, \theta)$ can be easily shown to be $A \lambda$. We can now compute type $\beta$ agent's excepted payoff from becoming corrupt when the discretion index to be used in the auction is $\lambda$ :

$$
E_{\theta}\left[\pi_{A}(\theta, \lambda)\right]-\beta=P \lambda-\mu B^{F}-\frac{\bar{\theta}+\underline{\theta}}{2} q_{C}-A \lambda-\mu B^{A}-\beta .
$$

Equating the right-hand side of (10) to 0 (the normalized payoff from being honest) and rearranging we get

$$
\beta^{*}=(P-A) \lambda-\mu\left(B^{F}+B^{A}\right)-\frac{\bar{\theta}+\underline{\theta}}{2} q_{C} .
$$

The following proposition is an immediate consequence of Eq. (11) and summarizes the main result of this section:

Proposition 6. Let Assumptions 1 and 2 be satisfied and let $\beta^{*}=(P-A) \lambda-$ $\mu\left(B^{F}+B^{A}\right)-(\bar{\theta}+\underline{\theta}) / 2 q_{C}$. Then, the probability the agent gets corrupted, $\gamma$, is

$$
\gamma= \begin{cases}\beta^{*} / \bar{\beta} & \text { if } \beta^{*}<\bar{\beta} \\ 1 & \text { otherwise }\end{cases}
$$

\section{Equilibrium}

Although we will omit a reference to it, in the rest of the paper we will implicitly make Assumptions 1 and 2. The following proposition provides the equilibrium values of discretion and the probability corruption.

Proposition 7. In equilibrium

$$
\begin{aligned}
& \hat{\lambda}=\frac{1+k}{2+\Gamma}, \\
& \hat{\gamma}=\frac{\Gamma-2 k}{2+\Gamma} .
\end{aligned}
$$




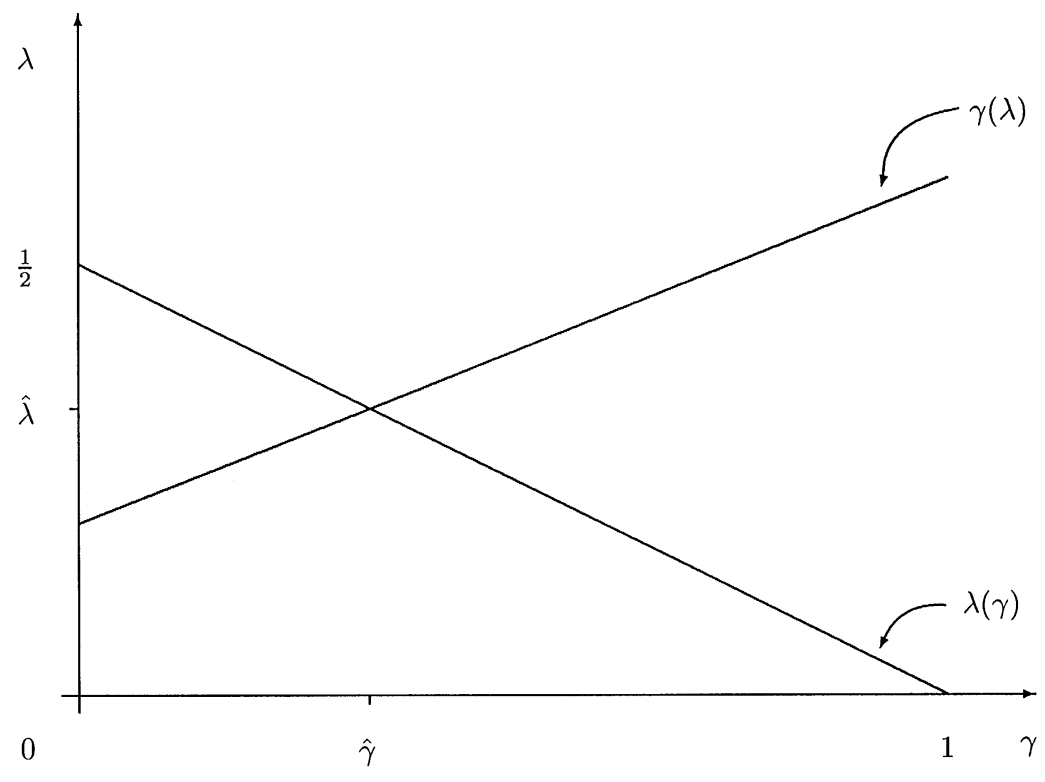

Fig. 1. Equilibrium.

Proof. Immediate from Propositions 5 and 6 and recalling the definition of $k$ and $\Gamma$ in Eqs. (8) and (9).

Fig. 1 summarizes the result by depicting the best response of the principal (parametrized by the discretion index $\lambda$ ) to a given probability for the agent to be corrupt, $\gamma$, and the best response to the agent (parametrized by the probability he gets corrupted, $\gamma$, prior to knowing his corruption cost, $\beta$ ) to a given discretion index $\lambda$. The intersection of the best responses provides equilibrium discretion and probability of corruption.

It is important to clarify at this point that, as was spelled out in Section 2, we are describing a situation in which the agent decides whether to be corrupt before the principal chooses the procurement mechanism and the principal decides on the procurement mechanism without observing whether the agent has decided to be corrupt. This implies in particular that the principal chooses the procurement mechanism based on his expectation of the decision to be corrupt which he considers to be given. With this setup the reason why the principal chooses a lower discretion index when he believes the agent is corrupt with probability $\gamma$ is that the expected marginal value of quality $q$ is decreasing in $\gamma$ as quality $q$ is only delivered with probability $1-\gamma$. rium.

The following proposition provides the comparative statics of the equilib- 
Proposition 8. The discretion index (probability of corruption) is increasing (decreasing) in $\mu, B^{F}, B^{A}, q_{C}$ and $\bar{\beta}$.

Proof. See the appendix.

Proposition 8 shows that the larger the penalty, the monitoring probability, the minimal quality to be provided under corruption, the lower the expected payoff of being corrupt and therefore, the lower the probability of corruption. On the other hand, it also shows that the larger the support of the matching cost, the larger is the average matching cost, the lower the expected payoff of being corrupt and, therefore, the lower the probability of corruption.

We believe that the fact that the comparative statics results presented in the Proposition 8 concord with common intuition provides support for the model we presented as a reasonable description of the way corruption decisions are taken in equilibrium and as a useful tool to study the relationship between corruption and competition. The next section turns to this analysis.

\section{Competition and corruption}

The goal of this section is to explore the relationship between corruption and competition. More specifically we will be interested in the impact on equilibrium corruption of the number of potential suppliers (procurement market competition) and of competition in the market for agents (procurement agents market competition).

\subsection{Competition in the procurement market and corruption}

In this subsection we want to study the impact of an increase in the competitiveness of the procurement market on the equilibrium. In the incomplete information setting proposed in this paper a natural exercise is to consider the impact of an increase in the number of potential suppliers. This can be shown to lead to lower markups in equilibrium bidding functions, lower expected profit for any type of firm, and lower expected equilibrium unit price.

Contrary to conventional wisdom, the next proposition shows that when competition among suppliers increases as a consequence of the increase of their number, corruption may increase. Proposition 9 in fact shows that corruption is increasing in competition for the given functional forms we have chosen. Since Proposition 9 does not generalize under the conditions in Section 2 of Che (1993), it should be stressed that we consider Proposition 9 as an effective way of demonstrating that increased competition in the procurement market does not necessarily imply lower corruption. 
Proposition 9. The discretion index (probability of corruption) is decreasing (increasing) in $N$.

Proof. See the appendix.

The proof of Proposition 9 is instructive in the sense that identifies the three forces that determine whether the ultimate impact on corruption is positive or negative. To do this we use the following result.

Lemma 1. For a given $\lambda$, the expected transfer to the winning firm is independent of $N$.

Proof. See the appendix.

Notice that Lemma 1 does not say that the price per unit of quality is constant in $N$. As a matter of fact the price per unit of quality is decreasing in $N$ as a consequence of both the lower expected minimum cost realization and of the lower informational rents implied by tougher competition.

By the same token, however, the lower expected unit cost of quality implies that the mechanism chosen by the principal in equilibrium will be such that the expected procured quality will be higher. Summarizing, while the lower expected unit cost tends to decrease the expected total transfer to the winning firm, the higher expected procured quality tends to increase the expected total transfer to the winning firm. With the functional forms and the distribution we have chosen, the two effects turn out to exactly cancel out each other to leave the expected total transfer to the winning firm as constant in $N$.

From Lemma 1 it should be clear that in the case we concentrate on, the net impact of a higher $N$ turns out to be the one implied by the reduction in firms' disagreement payoffs, $d_{F}(\lambda, \theta)$, which depends on the fact that the expected payoff if the agent behaves honestly is lower the higher $N$. Since this implies that the procurement agent appropriates a higher share of the surplus if he decides to be corrupt, being corrupt will be optimal for an agent with a higher cost $\beta$ and the probability of corruption will be higher.

The impact of the result in Lemma 1 is summarized in Fig. 2, in which it is shown that the agent's best response to any given discretion level, $\lambda$, shifts to the right, so that in the new equilibrium the probability of corruption will be higher $\left(\hat{\gamma}^{\prime}>\hat{\gamma}\right)$ and discretion lower $\left(\hat{\lambda}^{\prime}<\hat{\lambda}\right)$. It is interesting to note that while more competition (in the sense of a larger $N$ ) implies more corruption, it is also true that more corruption implies more competition (in the sense of lower expected payoffs for firms). Given a higher probability of corruption implies that the principal chooses a mechanism assigning lower weight to quality, the higher the probability of corruption, the more similar to each other firms will be, in the sense that firms with lower marginal costs of producing 


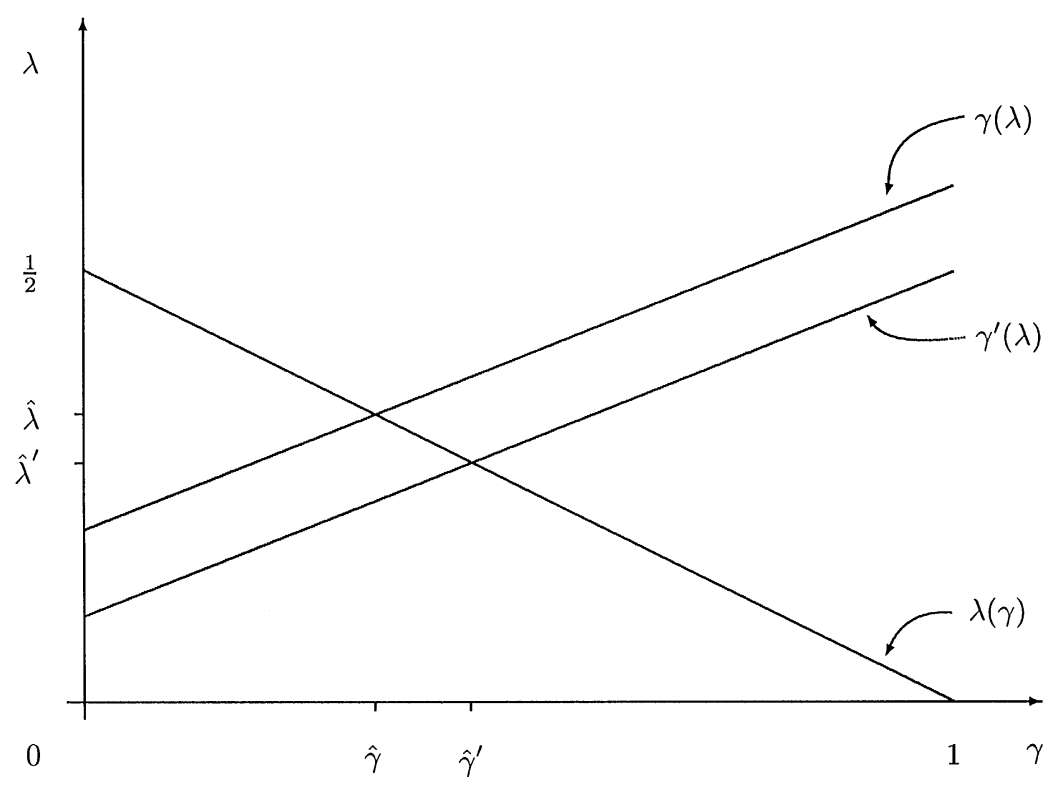

Fig. 2. Corruption with increased competition in the procurement market.

quality will have a lower advantage over firms with higher marginal costs. From Proposition 5 and Corollary 2 it is easy to see that, when corruption is higher, $\lambda$ is lower and the expected net transfer to any one firm is lower.

Although the result of Proposition 9 depends on the specified functional forms, it highlights the impact of larger numbers of firms when the principal reacts to the expected probability of corruption. In general we can see that the effects of a larger $N$ are the following:

1. The expected marginal cost of producing quality and the informational rents of the winning firm are lower, and the expected transfer per unit of quality to the winning firm is lower.

2. The lower expected marginal cost of producing quality implies a higher expected quality being supplied by the winning firm.

3. The expected payoff to a firm when the agent behaves honestly and therefore its disagreement payoff when bargaining with a corrupt agent is lower.

Notice that in general from 1 and 2 it is not possible to say whether the expected total transfer to the winning firm is higher or lower with a larger number of firms as the effects discussed in 1 and 2 tend to, respectively, decrease and increase the total transfer to the winning firm. This in turn implies 
that it is not possible to say whether tougher competition (parametrized by a higher $N$ ) leads to lower or higher corruption.

Lemma 1 on the other hand shows that, in the case we have concentrated on, the effects discussed in 1 and 2 exactly cancel out each other so that the sign of the net effect is determined by the effect discussed in 3 that implies that a larger number of firms decreases the reservation level of firms when they bargain with a corrupt agent and therefore leads to higher corruption.

Notice also that we only have considered the possibility of an exogenous penalty for the firm, $B^{F}$, but that it could be sensible to consider the impact of the possibility of excluding firms that have been found to be corrupt. Since this punishment is stronger the higher the expected equilibrium payoffs and therefore the lower the number of potential suppliers, it provides an additional reason why an increase in the number of potential suppliers may lead to an increase in corruption.

Before concluding this section we want to stress that, the fact that more competition may imply higher corruption does not necessarily imply that more competition is undesirable from the point of view of the principal. Since more corruption is harmful for the principal but increased competition is beneficial to him when the agent is honest, the comparison in general, is ambiguous.

\subsection{Competition in the market for procurement agents and corruption}

In the model we have presented, we assumed for the sake of simplicity that the procurement agent could verify procured quality at zero cost. Our results easily generalize to situations in which the procurement agent bears a cost of certifying that delivered quality is $q$ which is equal to $\eta q$, with $\eta>0$ observable. In this subsection we consider this kind of situation and we analyze how increased competition in the market for procurement agents affects corruption. To do this we make the following assumptions:

Assumption 3. If the firm that is awarded the realization of the project is supposed to provide quality $q$, the agent's cost of verifying quality is $\eta q$, regardless of whether the firm provided quality $q$ or quality $q_{C}$ (in the case the firm and the procurement agent reached a corrupt agreement).

Assumption 4. The procurement agent's ability, $1 / \eta$, is observable.

Assumption 5. Increased competition in the market for procurement agents implies that the employed procurement agent has higher ability (i.e., lower marginal cost of verifying procured quality).

Under Assumptions 3 and 4 it is easy to see that, when no corruption is possible, in equilibrium the firm with the lowest marginal cost of producing 
quality is assigned the realization of the project and it provides quality $q_{0}^{\prime}$ maximizing $V(q)-(2 \theta-\underline{\theta}) q-\eta q$. In other words, since higher procured quality implies higher verification costs, procured quality will be lower than in the case with zero verification costs.

Assumption 5 can be thought as representing a situation in which removing barriers to entry ${ }^{15}$ into employment in a public procurement agency implies that the average quality of employed procurement agents is higher while the salary is unchanged.

The next proposition states that stronger competition in the market for procurement agents implies higher corruption.

Proposition 10. Under Assumptions 3-5 the discretion index (probability of corruption) is lower (higher) the stronger the competition in the market for procurement agents.

\section{Proof. See the appendix.}

The intuition behind Proposition 10 is the following. Tougher competition in the market for procurement agents implies a higher ability of the employed procurement agent. Given the procurement agent has an observable higher ability, for any given probability of corruption, the optimal mechanism has to discount a lower additional cost of quality ( $\eta q$ is lower). Since this increases the profitability of corruption, the agent will be more inclined to corruption for any given $\lambda$. This is represented in Fig. 3, where the best response of the agent for two different values of $\eta$ is represented. Given $\eta_{1}<\eta_{0}$, the best response for $\eta_{1}$ lies to the right of the best response for $\eta_{0}$, and the associated equilibrium levels of discretion and the probability of corruption are such that $\hat{\lambda}_{1}<\hat{\lambda}_{0}$, and $\hat{\gamma}_{1}>\hat{\gamma}_{0}$, as claimed by Proposition 10. For comparison purposes, Fig. 3 also depicts the limit case in which $\eta=0$, which corresponds to the equilibrium analyzed in Section 5.

It is important to stress that, as in the previous case, an increase in competition in the market for procurement agent has an ambiguous effect on the principal's welfare: while he has to reimburse a lower cost to the agent and the optimal mechanism implies higher utility when the agent is honest, these effects may be outweighed by the higher probability of the agent being corrupt in equilibrium. ${ }^{16}$

The result of Proposition 10 is the same as the one proposed by Laffont and N'Guessan (1999) although the underlying reason is different. In our case,

\footnotetext{
${ }^{15}$ Examples include legal degree requirements, professional affiliations, or citizenship requirements.

${ }^{16}$ Notice that this ambiguity depends on the assumption that the procurement agent decides whether to be corrupt before the principal announces the allocation mechanism to be used.
} 


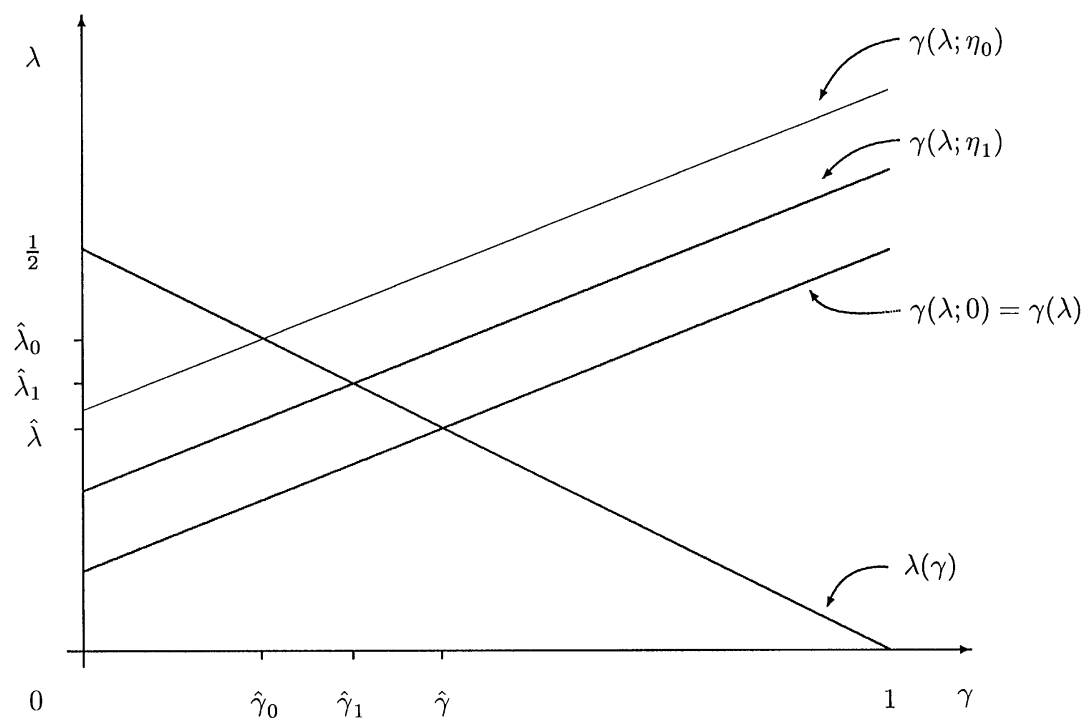

Fig. 3. Corruption with increased competition in the market for procurement agents.

when increased competition in the market for procurement agents selects a more efficient agent, the opportunity cost of reducing his discretion is higher. Since higher discretion implies higher profitability of corruption a higher probability of corruption ensues in equilibrium.

Before concluding, we want to consider the possibility that increased competition implies a lower wage for the procurement agent. Including wages in our model can be easily seen to deliver the standard result that a positive procurement wage differential with respect to wages in an alternative employment acts as a corruption deterrence. This in turn means that, if increased competition implies a lower wage differential, it then implies higher corruption, while if increased competition in the labor market at large implies lower wages, but unchanged wage differentials, no impact on corruption arises.

\section{Conclusions}

In this paper we pursued it has two different and related goals: To study the link between corruption and optimal procurement mechanisms and, taking advantage of the former, to explore the relationship between competition and corruption.

We showed that the possibility of corruption decreases the expected value of awarding a larger weight to quality and therefore of relying on the 
procurement agent's ability to verify procured quality. In other words, keeping into account the optimal regulatory response, the possibility for the agent to be corrupt implies that in equilibrium he is awarded less discretion.

Based on this we studied how corruption is affected by increased competition and in particular we asked the following questions:

1. What are the consequences of a larger number of potential suppliers in the procurement market?

2. What are the consequences of increased competition in the market for procurement agents?

Our analysis shows that it may well be that corruption is higher in a more competitive environment. This is not to say that increases in competition are not desirable, as in fact they may be beneficial, but to highlight that a cost associated with tougher competition may be higher corruption and that it is reasonable to expect that corruption and competition will grow together in certain markets.

\section{Acknowledgements}

Marco Celentani acknowledges the financial support of DGES of MEC (Spain) under projects UE95-0042 and PB98-00024 and of MURST (Italy) under project \# 9813282901_004. Juan-José Ganuza acknowledges the financial support of the Spanish Ministry of Science and Technology under project BEC2000-1026. We wish to thank Maria Angeles de Frutos, the Editor, Klaus Schmidt, two anonymous referees, and the participants to the Workshop on Financial Markets, Legal System and Judicial Enforcement, Università di Napoli Federico II and Games 2000, Bilbao, for very useful discussions and suggestions. All remaining errors are ours.

\section{Appendix}

Proof of Proposition 1. By the Revelation Principle we can restrict without loss of generality to direct revelation mechanisms. Let $q(\theta), p(\theta), \sigma(\theta)$ denote respectively the vectors denoting quality levels, transfers, and probability of being awarded the project of each one of the $N$ firms. Let, $i=1, \ldots, N$,

$$
v_{i}\left(\sigma_{i}\left(\theta_{i}, \theta_{-i}\right), q_{i}\left(\theta_{i}, \theta_{-i}\right) ; \theta_{i}\right)=\sigma_{i}\left(\theta_{i}, \theta_{-i}\right) \theta_{i} q_{i}\left(\theta_{i}, \theta_{-i}\right)
$$

and let

$$
\mathscr{V}_{i}\left(\theta_{i}^{\prime} ; \theta_{i}\right)=E_{\theta_{-i}}\left[p_{i}\left(\theta_{i}^{\prime}, \theta_{-i}\right)-v_{i}\left(\sigma_{i}\left(\theta_{i}^{\prime}, \theta_{-i}\right), q_{i}\left(\theta_{i}^{\prime}, \theta_{-i}\right) ; \theta_{i}\right)\right] .
$$


The optimal mechanism is then a solution to the following program:

$$
\max _{q(\theta), p(\theta), \sigma(\theta)} E_{\theta}\left[\sum_{i=1}^{N} \sigma_{i}(\theta) \log q_{i}(\theta)-\sum_{i=1}^{N} p_{i}(\theta)\right]
$$

s.t.

$$
\begin{aligned}
& \mathscr{V}_{i}\left(\theta_{i} ; \theta_{i}\right) \geqslant 0, \quad \forall \theta_{i} \in[\underline{\theta}, \bar{\theta}], \quad \forall i \in N, \\
& \mathscr{V}_{i}\left(\theta_{i} ; \theta_{i}\right) \geqslant \mathscr{V}_{i}\left(\theta_{i}^{\prime} ; \theta_{i}\right), \quad \forall \theta_{i}, \theta_{i}^{\prime} \in[\underline{\theta}, \bar{\theta}], \quad \forall i \in N, \\
& \sigma_{i}(\theta) \geqslant 0 \quad \text { and } \sum_{i=1}^{N} \sigma_{i}(\theta) \leqslant 1, \quad \forall \theta \in[\underline{\theta}, \bar{\theta}] .
\end{aligned}
$$

Let

$$
U_{i}\left(\theta_{i}\right)=\mathscr{V}_{i}\left(\theta_{i} ; \theta_{i}\right)
$$

then we can rewrite the principal's objective function as

$$
R=E_{\theta}\left[\sum_{i=1}^{N} \sigma_{i}(\theta) \log q_{i}(\theta)-\sum_{i=1}^{N} v_{i}\left(\sigma_{i}(\theta), q_{i}(\theta) ; \theta_{i}\right)\right]-\sum_{i=1}^{N} E_{\theta_{i}}\left[U_{i}\left(\theta_{i}\right)\right] .
$$

From the envelope theorem

$$
\begin{aligned}
\frac{\mathrm{d} U_{i}}{\mathrm{~d} \theta_{i}} & =-E_{\theta_{-i}}\left[\frac{\partial}{\partial \theta_{i}} v_{i}\left(\sigma_{i}\left(\theta_{i}, \theta_{-i}\right), q_{i}\left(\theta_{i}, \theta_{-i}\right) ; \theta_{i}\right)\right] \\
& =-E_{\theta_{-i}}\left[\sigma_{i}\left(\theta_{i}, \theta_{-i}\right) q_{i}\left(\theta_{i}, \theta_{-i}\right)\right]
\end{aligned}
$$

and integrating we get

$$
U_{i}\left(\theta_{i}\right)=U_{i}(\bar{\theta})+\int_{\theta_{i}}^{\bar{\theta}} E_{\theta_{-i}}\left[\sigma_{i}\left(\tilde{\theta}_{i}, \theta_{-i}\right) q_{i}\left(\tilde{\theta}_{i}, \theta_{-i}\right)\right] \mathrm{d} \tilde{\theta}_{i} .
$$

Since the principal's utility is decreasing in $U_{i}(\bar{\theta})$, at the optimum $U_{i}(\bar{\theta})=0$. Then from (A.5)

$$
E_{\theta_{i}}\left[U_{i}\left(\theta_{i}\right)\right]=\int_{\underline{\theta}}^{\bar{\theta}}\left[\int_{\theta_{i}}^{\bar{\theta}} E_{\theta_{-i}}\left[\sigma_{i}\left(\tilde{\theta}_{i}, \theta_{-i}\right) q_{i}\left(\tilde{\theta}_{i}, \theta_{-i}\right)\right] \mathrm{d} \tilde{\theta}_{i}\right] f\left(\theta_{i}\right) \mathrm{d} \theta_{i} .
$$

Integrating by parts,

$$
\begin{aligned}
E_{\theta_{i}}\left[U_{i}\left(\theta_{i}\right)\right]= & {\left[\left[\int_{\theta_{i}}^{\bar{\theta}} E_{\theta_{-i}}\left[\sigma_{i}\left(\tilde{\theta}_{i}, \theta_{-i}\right) q_{i}\left(\tilde{\theta}_{i}, \theta_{-i}\right)\right] \mathrm{d} \tilde{\theta}_{i}\right] F\left(\theta_{i}\right)\right]_{\underline{\theta}}^{\bar{\theta}} } \\
& +\int_{\underline{\theta}}^{\bar{\theta}} E_{\theta_{-i}}\left[\sigma_{i}\left(\tilde{\theta}_{i}, \theta_{-i}\right) q_{i}\left(\theta_{i}, \theta_{-i}\right)\right] F\left(\theta_{i}\right) \mathrm{d} \theta_{i} .
\end{aligned}
$$


Since

$$
\left[\left[\int_{\theta_{i}}^{\bar{\theta}} E_{\theta_{-i}}\left[\sigma_{i}\left(\tilde{\theta}_{i}, \theta_{-i}\right) q_{i}\left(\tilde{\theta}_{i}, \theta_{-i}\right)\right] \mathrm{d} \tilde{\theta}_{i}\right] F\left(\theta_{i}\right)\right]_{\underline{\theta}}^{\bar{\theta}}=0
$$

multiplying and dividing by the density function $f\left(\theta_{i}\right)$, we obtain

$$
\begin{aligned}
E_{\theta_{i}}\left[U_{i}\left(\theta_{i}\right)\right] & =\int_{\underline{\theta}}^{\bar{\theta}} E_{\theta_{-i}}\left[\sigma_{i}\left(\theta_{i}, \theta_{-i}\right) q_{i}\left(\theta_{i}, \theta_{-i}\right)\right] \frac{F\left(\theta_{i}\right)}{f\left(\theta_{i}\right)} f\left(\theta_{i}\right) \mathrm{d} \theta_{i} \\
& =E_{\theta}\left[\sigma_{i}\left(\theta_{i}, \theta_{-i}\right) q_{i}\left(\theta_{i}, \theta_{-i}\right) \frac{F\left(\theta_{i}\right)}{f\left(\theta_{i}\right)}\right]
\end{aligned}
$$

and substituting $F\left(\theta_{i}\right) / f\left(\theta_{i}\right)=\left(\theta_{i}-\underline{\theta}\right)$ into the above expression we obtain

$$
E_{\theta_{i}}\left[U_{i}\left(\theta_{i}\right)\right]=E_{\theta}\left[\left(\theta_{i}-\underline{\theta}\right) \sigma_{i}(\theta) q_{i}(\theta)\right] .
$$

Substituting (A.1) and (A.6) into (A.4) and after straightforward computations we get

$$
R=E_{\theta}\left[\sum_{i=1}^{N} \sigma_{i}(\theta) \log q_{i}(\theta)-\sum_{i=1}^{N}\left(2 \theta_{i}-\underline{\theta}\right) q_{i}(\theta) \sigma_{i}(\theta)\right] .
$$

Sufficient conditions for incentive compatibility condition (A.2) to hold are that (A.5) holds and that $\Sigma_{i}\left(\theta_{i}\right)=-E_{\theta_{-i}}\left[\sigma_{i}\left(\theta_{i}, \theta_{-i}\right) q_{i}\left(\theta_{i}, \theta_{-i}\right)\right]$ is nonincreasing. Hence the optimal mechanism solves

$$
\max _{q(\theta), \sigma(\theta)} E_{\theta}\left[\sum_{i=1}^{N} \sigma_{i}(\theta) \log q_{i}(\theta)-\sum_{i=1}^{N}\left(2 \theta_{i}-\underline{\theta}\right) q_{i}(\theta) \sigma_{i}(\theta)\right]
$$

s.t.

$$
\sigma_{i}(\theta) \geqslant 0 \quad \text { and } \quad \sum_{i=1}^{N} \sigma_{i}(\theta) \leqslant 1, \quad \forall \theta \in[\underline{\theta}, \bar{\theta}]
$$

$\Sigma_{i}(\cdot)$ nonincreasing.

Maximize (A.7) ignoring the incentive compatibility constraint. This yields $\sigma_{i}(\theta)=1$ if and only if $\theta_{i}=\min _{j \in N} \theta_{j}$ and $q_{i}\left(\theta_{i}, \theta_{-i}\right) \in \arg \max _{q_{i}}\left\{\log q_{i}-\right.$ $\left.\left(2 \theta_{i}-\underline{\theta}\right) q_{i}\right\} \Leftrightarrow q_{i}=1 /\left(2 \theta_{i}-\underline{\theta}\right)<1 / \theta_{i}$ so that the quality level is ex-post inefficient. Given the optimal quality $q_{i}\left(\theta_{i}, \theta_{-i}\right)$ and the optimal probability of assignment $\sigma_{i}\left(\theta_{i}, \theta_{-i}\right)$ are nonincreasing, $\Sigma_{i}(\cdot)$ is nonincreasing and the incentive compatibility constraint is satisfied.

Proof of Corollary 2. (1) To obtain the claim it suffices to show that in a first-score auction using $\tilde{S}(\lambda, q, p)$ as the score function, the firm with the lowest $\theta$ wins with probability 1 and to then compute expected winning price and quality it is sufficient to compute the expected quality and price 
using the distribution of the minimum of an $N$-dimensional sample from $F(\theta)=(\theta-\underline{\theta}) /(\bar{\theta}-\underline{\theta})$. To show this replace the equilibrium offer in the score function to obtain

$$
\begin{aligned}
S(\lambda, \theta) & =\tilde{S}\left(\lambda, q_{\mathrm{E}}(\theta, \lambda), p_{\mathrm{E}}(\theta, \lambda)\right) \\
& =\lambda \log \frac{2 \lambda}{2 \theta-\underline{\theta}}+\frac{\underline{\theta}}{2} \frac{2 \lambda}{2 \theta-\underline{\theta}}-\frac{2 \lambda \theta}{2 \theta-\underline{\theta}}-\int_{\theta}^{\bar{\theta}}\left(\frac{\bar{\theta}-s}{\bar{\theta}-\theta}\right)^{N-1} \frac{2 \lambda}{2 s-\underline{\theta}} \mathrm{d} s \\
& =\lambda \log \frac{2 \lambda}{2 \theta-\underline{\theta}}-\lambda-\int_{\theta}^{\bar{\theta}}\left(\frac{\bar{\theta}-s}{\bar{\theta}-\theta}\right)^{N-1} \frac{2 \lambda}{2 s-\underline{\theta}} \mathrm{d} s .
\end{aligned}
$$

Using Leibniz's rule to differentiate $S(\lambda, \theta)$ in Eq. (21) with respect to $\theta$ we get

$$
\begin{aligned}
\frac{\partial S(\lambda, \theta)}{\partial \theta}= & -\frac{2 \lambda}{2 \theta-\underline{\theta}}+\lambda\left(\frac{\bar{\theta}-\theta}{\bar{\theta}-\theta}\right)^{N-1} \frac{2}{2 \theta-\underline{\theta}} \\
& -\lambda \int_{\theta}^{\bar{\theta}}(N-1)\left(\frac{\bar{\theta}-s}{\bar{\theta}-\theta}\right)^{N-2} \frac{\bar{\theta}-s}{(\bar{\theta}-\theta)^{2}} \frac{2}{2 s-\underline{\theta}} \mathrm{d} s \\
= & -\lambda \int_{\theta}^{\bar{\theta}}(N-1)\left(\frac{\bar{\theta}-s}{\bar{\theta}-\theta}\right)^{N-2} \frac{\bar{\theta}-s}{(\bar{\theta}-\theta)^{2}} \frac{2}{2 s-\underline{\theta}} \mathrm{d} s<0
\end{aligned}
$$

which implies that the score assigned to firms with lower marginal costs of producing quality is higher and concludes the proof of the first part. (2) immediate from (1) and Proposition 2.

Proof of Proposition 4. The optimal mechanism with corruption is the solution to

$$
\max _{q(\theta), p(\theta), \sigma(\theta)}(1-\gamma) E_{\theta}\left[\sum_{i=1}^{N} \sigma_{i}(\theta) \log q_{i}(\theta)-\sum_{i=1}^{N} p_{i}(\theta)\right]+\gamma\left(\log q_{C}-p_{C}\right)
$$

s.t.

$$
\begin{aligned}
& \mathscr{V}_{i}\left(\theta_{i} ; \theta_{i}\right) \geqslant 0, \quad \forall \theta_{i} \in[\underline{\theta}, \bar{\theta}], \quad \forall i \in N, \\
& \mathscr{V}_{i}\left(\theta_{i} ; \theta_{i}\right) \geqslant \mathscr{V}_{i}\left(\theta_{i}^{\prime} ; \theta_{i}\right), \quad \forall \theta_{i}, \theta_{i}^{\prime} \in[\underline{\theta}, \bar{\theta}], \quad \forall i \in N, \\
& \sigma_{i}(\theta) \geqslant 0 \quad \text { and } \sum_{i=1}^{N} \sigma_{i}(\theta) \leqslant 1, \quad \forall \theta \in[\underline{\theta}, \bar{\theta}] .
\end{aligned}
$$


Since by Assumption 1 the maximand is equal to

$$
E_{\theta}\left[(1-\gamma) \sum_{i=1}^{N} \sigma_{i}(\theta) \log q_{i}(\theta)-\sum_{i=1}^{N} p_{i}(\theta)\right]+\gamma \log q_{C}
$$

the problem is identical to the one without corruption with the only difference that $\log q$ is replaced by $(1-\gamma) \log q$ and the solution is the one given in Proposition 4.

Proof of Proposition 8. From $\hat{\lambda}=(1+k) /(2+\Gamma)$, we get $\mathrm{d} \hat{\lambda} / \mathrm{d} a=\partial \hat{\lambda} / \partial k \partial k / \partial a$ $>0$ for all $a \in\left\{B_{A}, B_{F}, \mu, w, q_{C}\right\}$, given $\partial \hat{\lambda} / \partial k>0$ and $\partial k / \partial a>0$. From

$$
\hat{\gamma}=\frac{\Gamma-2 k}{2+\Gamma}=\frac{(P-A)-2\left[\frac{\bar{\theta}+\underline{\theta}}{2} q_{C}+\mu\left(B^{F}+B^{A}\right)\right]}{2 \bar{\beta}+(P-A)},
$$

we get $\mathrm{d} \hat{\gamma} / \mathrm{d} \bar{\beta}<0$.

Proof of Lemma 1. Since from Corollary 2 the expected procurement price (expected transfer to the winning firm) is $p^{*}(\lambda)=P \lambda$, it is sufficient to show that $\partial P / \partial N=0$.

From Proposition 3 when $\lambda=\frac{1}{2}$ the first-score auction implements the optimal procurement mechanism without corruption. Thus the principal's expected surplus has to be the same in both mechanisms. Recalling we denote by $\theta_{1}=\min \left\{\theta_{i}\right\}_{i=1}^{N}$, the first-order statistic of $\theta$, the optimal mechanism gives the principal the following expected surplus:

$$
W^{*}=E_{\theta_{1}}\left[\log q_{0}\left(\theta_{1}\right)-\left(2 \theta_{1}-\underline{\theta}\right) q_{0}\left(\theta_{1}\right)\right] .
$$

The first-score auction gives the principal the following expected surplus:

$$
W_{\mathrm{FS}}=E_{\theta_{1}}\left[\log q_{\mathrm{E}}\left(\theta_{1}, \frac{1}{2}\right)-p_{\mathrm{E}}\left(\theta_{1}, \frac{1}{2}\right)\right]
$$

where

$$
\begin{aligned}
& q_{\mathrm{E}}\left(\theta_{i}, \frac{1}{2}\right)=\frac{1}{2 \theta_{i}-\underline{\theta}}, \\
& p_{\mathrm{E}}\left(\theta_{i}, \frac{1}{2}\right)=\frac{\theta_{i}}{2 \theta_{i}-\underline{\theta}}+\int_{\theta_{i}}^{\bar{\theta}}\left[\frac{\bar{\theta}-s}{\bar{\theta}-\theta_{i}}\right]^{N-1} \frac{1}{2 s-\underline{\theta}} \mathrm{d} s .
\end{aligned}
$$

From $W^{*}=W_{\mathrm{FS}}$ and $q_{0}\left(\theta_{i}\right)=q_{\mathrm{E}}\left(\theta_{i}, \frac{1}{2}\right)$, we obtain

$$
E_{\theta_{1}}\left[\left(2 \theta_{1}-\underline{\theta}\right) q_{0}\left(\theta_{1}\right)\right]=E_{\theta_{1}}\left[p_{\mathrm{E}}\left(\theta_{1}, \frac{1}{2}\right)\right]=\frac{1}{2} P .
$$


Substituting $q_{0}\left(\theta_{1}\right)=1 /\left(2 \theta_{1}-\underline{\theta}\right)$, we get

$$
E_{\theta_{1}}\left[\left(2 \theta_{1}-\underline{\theta}\right) \frac{1}{2 \theta_{1}-\underline{\theta}}\right]=1=\frac{1}{2} P
$$

and $P$ is shown to be independent of $N$.

Proof of Proposition 9. Since $\hat{\gamma}=(\Gamma-2 k) /(2+\Gamma)$ we have $\mathrm{d} \hat{\gamma} / \mathrm{d} N=(\partial \hat{\gamma} /$ $\partial \Gamma)(\partial \Gamma / \partial N)$. Notice that $\partial \hat{\gamma} / \partial \Gamma>0$. Moreover since $\partial P / \partial N=0$ and $\partial A / \partial N$ $<0$, from $\Gamma=(1 / \bar{\beta})[(P-A)]$ we get $\partial \Gamma / \partial N>0$ which together with $\partial \hat{\gamma} / \partial \Gamma$ $>0$ implies $\mathrm{d} \hat{\gamma} / \mathrm{d} N>0$.

Proof of Proposition 10. Under Assumptions 3 and 4 the equilibrium expressions are the same as (12) and (13) with the only difference that the constant $\Gamma$ is replaced by

$$
\begin{aligned}
\Gamma^{\prime}= & \frac{1}{\bar{\beta}} \int_{\underline{\theta}}^{\bar{\theta}}\left[\frac{2 \theta}{2 \theta+\eta-\underline{\theta}}+\int_{\theta}^{\bar{\theta}}\left(\frac{N-1}{N}\right)\left(\frac{\bar{\theta}-s}{\bar{\theta}-\theta}\right)^{N-1} \frac{2}{2 s+\eta-\underline{\theta}} \mathrm{d} s\right] \\
& \times \frac{N}{\bar{\theta}-\underline{\theta}}\left(\frac{\bar{\theta}-\theta}{\bar{\theta}-\underline{\theta}}\right)^{N-1} \mathrm{~d} \theta .
\end{aligned}
$$

Since it is easy to check that $\Gamma^{\prime}$ is decreasing in $\eta$, and the equilibrium probability of corruption $\hat{\gamma}^{\prime}$ is increasing in $\Gamma^{\prime}$, it is immediate to recognize that under Assumption 5 corruption is increasing in the competition in the market for procurement agents.

\section{References}

Ades, A., Di Tella, R., 1997. National champions and corruption: Some unpleasant interventionist arithmetic. Economic Journal 107, 1023-1042.

Ades, A., Di Tella, R., 1999. Rents, competition, and corruption. American Economic Review 89 (4), 982-993.

Banfield, E.C., 1975. Corruption as a feature of governmental organization. Journal of Law and Economics 58, 587-605.

Beck, P., Maher, M., 1986. A comparison of bribery and bidding in thin markets. Economics Letters 20, 1-5.

Bliss, C., Di Tella, R., 1997. Does competition kill corruption? Journal of Political Economy 105, 1001-1023.

Branco, F., 1997. The design of multidimensional auctions. RAND Journal of Economics 28, 63-81.

Celentani, M., Ganuza, J.-J., 1999. Corruption and the Hadleyburg effect, Working Paper No. 382, Department of Economics, Universitat Pompeu Fabra.

Celentani, M., Ganuza, J.-J., 2001. Organized vs. competitive corruption, Working Paper No. 526, Department of Economics, Universitat Pompeu Fabra. 
Che, Y., 1993. Design competition through multidimensional auctions. RAND Journal of Economics 24, 668-680.

Laffont, J.-J., N'Guessan, T., 1999. Competition and corruption in an agency relationship. Journal of Development Economics 60, 271-295.

Laffont, J.-J., Tirole, J., 1987. Auctioning incentive contracts. Journal of Political Economy 95 , 921-937.

Laffont, J.-J., Tirole, J., 1991. Auction design and favoritism. International Journal of Industrial Organization 9, 9-42.

Lien, D., 1986. A note on competitive bribery games. Economics Letters 22, 337-341.

Lien, D., 1987. Asymmetric information in competitive bribery games. Economics Letters 23, $153-156$.

McAfee, R.P., McMillan, J., 1987. Competition for agency contracts. RAND Journal of Economics 18, 296-307.

Rose-Ackerman, S., 1996. Redesigning the state to fight corruption. Public Policy for Private Sector, World Bank.

Riordan, M.H., Sappington, D.E.M., 1987. Awarding monopoly franchises. American Economic Review 77, 375-387.

Wei, S.-J., 2000. Natural openness and good government. Working Paper No. W7765, NBER, USA. 